\title{
An Architecture for Learning Agents
}

\author{
Bartłomiej Śnieżyński \\ AGH University of Science and Technology, Institute of Computer Science \\ Kraków, Poland \\ Bartlomiej.Sniezynski@agh.edu.pl
}

\begin{abstract}
This paper contains a proposal of an architecture for learning agents. The architecture supports centralized learning. Learning may be performed by several agents in the system, but it should be independent (without communication or cooperation connected with the learning process). An agent may have several learning modules for different aspects of its activity. Each module can use different learning strategy. Application of the architecture is studied on example of Fish-Banks game simulator.
\end{abstract}

Keywords: multi-agent systems, machine learning, agent architecture.

\section{Introduction}

Multi-agent systems often work in complex environments. Therefore it is very difficult (or sometimes impossible) to specify and implement all system details a priori.

Applying learning algorithms allows to overcome such problems. One can implement an agent that is not perfect, but improves its performance. This is why machine learning term appears in a context of agent systems for several years.

A lot of multi-agent systems, which are able to learn, have been built so far. But in these works authors use their own architectures for learning agents, specialized for the considered application domains. The universal model of the learning agent was missing. It should be general enough to use in every domain, cover as many learning methods as possible, but also it should be specific enough to help to develop learning multi-agent systems.

This paper contains a proposal of the architecture for learning agents. The proposed architecture supports centralized learning only. It means that all the learning process is performed by an agent itself. Learning may be performed by several agents in the system, but it should be independent (without communication or cooperation with other agents regarding the learning process).

In the following sections learning in multi agent systems is briefly discussed, the architecture of the learning agent is described, and its use in developed system is presented.

\section{Learning in Multi-agent Systems}

Machine learning focuses mostly on research on an isolated process performed by only one module in the whole system. The multi-agent approach concerns the

M. Bubak et al. (Eds.): ICCS 2008, Part III, LNCS 5103, pp. 722 730, 2008.

(C) Springer-Verlag Berlin Heidelberg 2008 
systems composed of autonomous elements, called agents, whose actions lead to the realization of given goals. In this context, learning is based on the observation of the influences of activities, performed to achieve the goal by an agent itself or by other agents. Learning may proceed in a traditional - centralized (one learning agent) or decentralized manner. In the second case more than one agent is engaged in one learning process.

In multi-agent systems the most common technique is reinforcement learning [1]. It allows to generate a strategy for an agent in a situation, when the environment provides some feedback after the agent has acted. Feedback takes the form of a real number representing reward, which depends on the quality of the action executed by the agent in a given situation. The goal of the learning is to maximize estimated reward.

Supervised learning is not so widely used in multi-agent systems. However there are some works using such strategies (e.g. 23]). Supervised learning allows to generate knowledge from examples. Using this method instead of reinforcement learning has several advantages, see [4.

Architecture for learning agent can be found in [5]. Unfortunately it fits mainly reinforcement learning.

\section{The Learning Agent Architecture}

In this paper we propose a learning agent architecture for centralized learning, which allows to use several learning modules in an agent. The architecture is presented in Fig. 1

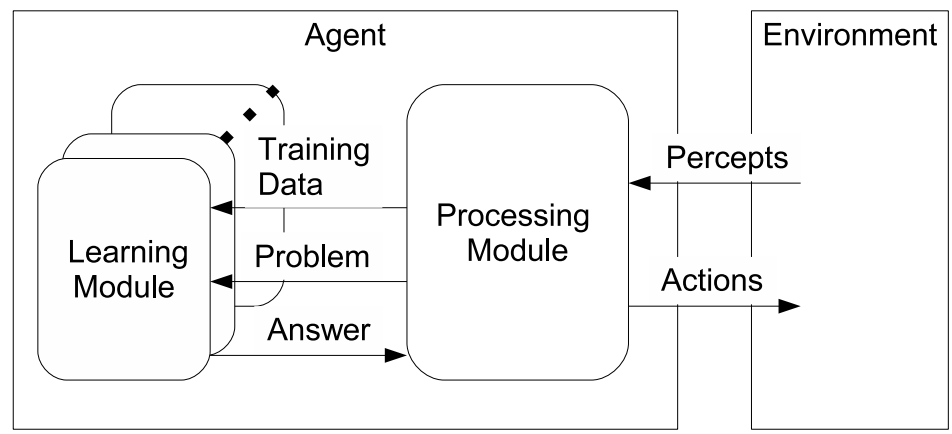

Fig. 1. Learning agent architecture

An agent gets percepts from an environment, and executes actions to interact with the environment. The main unit in an agent is a processing module, which is responsible for analyzing sensor input, and choosing appropriate action. It can be realized in a simple way, e.g. using reactive agent architecture, or in a complex way, e.g. using layered or BDI architectures. To improve the performance, agent 
can use learning modules. To learn, agent should provide a training data. After learning, the module can be used to get an answer for a provided problem. Therefore, the training data and the problem are inputs for the module, and the answer is an output. Several learning modules, using various learning strategies, can be used by one agent for different aspects of its activity.

Characteristics of the training data, the problem and the answer depend on the learning strategy used in the module. Therefore we can define a learning module as a four-tuple: (Learning strategy, Training data, Problem, Answer).

Details of the learning modules are domain-specific. Learning strategy, (knowledge representation, learning algorithm, and conditions for which learning is executed), structure and source of Training data, Problem, and Answer should be carefully designed by the system architect. Additional research is necessary to provide guidance in this aspect.

Two types of learning modules were developed and tested so far: reinforcement learning module and inductive rule learning (see section 4). Although, other learning methods can be also used. Below modules for three types of popular learning strategies are characterized.

\subsection{Reinforcement Learning}

As it was mentioned earlier, the most popular learning method in multi-agent systems is reinforcement learning. In this method, an agent gets description of the current state and using its current strategy chooses an appropriate action from a defined set. Next, using reward from the environment and next state description it updates its strategy. Several methods of choosing the action and updating the strategy have been developed so far. E.g. in Q-learning developed by Chris Watkins [6] action with the highest predicted value $(Q)$ is chosen. $Q$ is a function that estimates value of the action in a given state:

$$
Q: A \times X \rightarrow \Re,
$$

where $A$ is a set of actions, and $X$ is a set of possible states. $Q$ function is updated after action execution:

$$
\begin{array}{r}
Q(a, x):=Q(a, x)+\beta \Delta \\
\Delta=\gamma Q_{\max }+r-Q(a, x) \\
Q_{\max }=\max _{a} Q\left(a, x^{\prime}\right)
\end{array}
$$

where $x, x^{\prime} \in X$ are subsequent states, $a \in A$ is an action chosen, $r$ is a reward obtained from the environment, $\gamma \in[0,1]$ is a discount rate (importance of the future rewards), and $\beta \in(0,1)$ is a learning rate. Various techniques are used to prevent from getting into a local optimum. The idea is to explore the solution space better by choosing not optimal actions (e.g. random or not performed in a given state yet) from time to time.

Reinforcement learning module can be responsible for managing all the agent activities or only a part of it (it can be activated in some type of states or can be 
responsible for selected actions only). The Problem definition that is provided consists of the description of the current state. The Answer is an action chosen using the current strategy (current $Q$ function). Training data consists of the next state description (after executing action returned by the module), and a reward. The reward may be observed by the agent or may be calculated by the processing module using some performance measures.

\subsection{Supervised Learning}

Supervised learning allows to generate an approximation of a function $f: X \rightarrow C$ from labeled examples, which consist of pairs of arguments and function values. This approximation is called a hypothesis $h$. Elements of $X$ are described by set of attributes $A=\left(a_{1}, a_{2}, \ldots, a_{n}\right)$, where $a_{i}: X \rightarrow D_{i}$. Therefore $x^{A}=$ $\left(a_{1}(x), a_{2}(x), \ldots, a_{n}(x)\right)$ is used instead of $x$.

Supervised learning module gets a Training data, which is a set $\left\{\left(x^{A}, f(x)\right)\right\}$, and generates hypothesis $h$. Problem is a $x^{A}$, and the Answer is $h\left(x^{A}\right)$.

There are lots of supervised learning methods. They use various hypothesis representation, and various methods of hypothesis construction. One of the most popular algorithms is $\mathrm{C} 4.5$, inductive decision tree learning algorithm developed by Ross Quinlan [7. It can be used if the size of the set $C$ is small. In such a case we call $C$ a set of classes, and hypothesis is called a classifier. C4.5 uses decision trees to represent $h$. The basic idea of learning is as follows. The tree is learned from examples recursively. If (almost) all examples in the training data belong to one class, the tree consisting of the leaf labeled by this class is returned. In the other case, the best attribute for the test in the root is chosen (using entropy measure), training examples are divided according to the selected attribute values, and the procedure is called recursively for every attribute test result with the rest of attributes and appropriate examples as parameters.

Another learning algorithm with broad range of abilities, which was used in the implemented system (see section 4) is AQ. It was developed by Ryszard Michalski [8]. Its subsequent versions are still developed. This algorithm also generates classifier from the training data, but $h$ is represented by a set of rules, which have tests on attribute values in the premise part, and a class in a conclusion. Rules are generated using sequential covering: the best rule (e.g. giving a good answer for the most examples) is constructed by a beam search, examples covered by this rule are eliminated from a training set, and the procedure repeats.

Other methods, using different knowledge representation, such as support vector machines, Bayesian or instance-based models also fit the above specification. Similarly, learning module using artificial neural networks for classification or function approximation have the same input and output.

What is important, in the case of supervised learning, the processing module should provide in the training data a proper function value $f(x)$ for examples. If we are not able to provide this, inductive learning can not be used. However, if we have at least some qualitative information about $f(x)$ for given $x^{A}$, as we suggested in [9] we can build a classifier. Details of this work-around can be found in section 4.2 


\subsection{Unsupervised Learning}

In unsupervised learning the task of the learning module is to organize examples into groups called clusters, whose members are similar in a some way. Examples of this strategy are Kohonen neural networks and clustering. Training data have a form of example descriptions: $\left\{x^{A}\right\}$ (without any label). The Problem is an example description $x^{A}$, and the Answer is the example's cluster identifier.

This type of module was not tested yet. It is presented here to show that the framework proposed is general enough to cover this type of learning.

\section{Application of the Architecture}

In this section we present application of the proposed learning agent architecture. An multi agent system was built to simulate the Fish Banks game [10. The game is a dynamic environment providing resources, action execution procedures, and time flow represented by game rounds. Each round consists of the following steps: ships and money update, ship auctions, trading session, ship orders, ship allocation, fishing, fish number update.

Agents represent players that manage fishing companies. Each company aims at collecting maximum assets expressed by the amount of money deposited at a bank account and the number of ships. The company earns money by fishing at fish banks. The environment provides two fishing areas: coastal and deep-sea. Agents can also keep their ships at the port. The cost of deep-sea fishing is the highest. The cost of staying at the port is the lowest but such ship does not catch fish. Initially, it is assumed that the number of fish in both banks is close to the bank's maximal capacity. Therefore, at the beginning of game deep-sea fishing is more profitable.

Usually, exploration of the banks by fishing is too high and after several rounds the number of fish decreases to zero. It is a standard case of "the tragedy of commons" [11. It is more reasonable to keep ships at the harbor then, therefore companies should change theirs strategies.

Agents may observe the following aspects of the environment: arriving of new ships bought from a shipyard, money earned in the last round, ships allocations of all agents, and fishing results for deep sea and inshore area. All types of agents can execute the following two types of actions: order ships, allocate ships.

Three types of agents can play the game in the system: two types of learning agents using reinforcement learning and rule inductive learning, and a random agent.

Order ships action is currently very simple. It is implemented in all types of agents in the same way. At the beginning of the game every agent has 10 ships. Every round, if it has less than 15 ships, there is $50 \%$ chance that it orders two new ships.

Ships allocation action is controlled by a learning module or is done randomly. It is based on the method used in [12. The allocation action is represented by a triple $(h, d, c)$, where $h$ is the number of ships left in a harbor, $d$ and $c$ are numbers of ships sent to a deep sea, and a coastal area respectively. Agents 
generate a list of allocation strategies for $h=0 \%, 25 \%, 50 \%, 75 \%$, and $100 \%$ of ships that belong to the agent. The rest of ships $(r)$ is partitioned; for every $h$ the following candidates are generated:

1. All: $(h, 0, r),(h, r, 0)$ - send all remaining ships to a deep sea or coastal area,

2. Check: $(h, 1, r-1),(h, r-1,1)$ - send one ship to a deep sea or coastal area and the rest to the other,

3. Three random actions: $(h, x, r-x)$, where $1 \leq x<r$ is a random number allocate remaining ships in a random way,

4. Equal: $(h, r / 2, r / 2)$ - send equal number of ships to both areas.

The random agent allocates ships using one of the candidates chosen by random. Methods used by learning agents and their learning modules are described below.

\subsection{Reinforcement Learning Agent}

Reinforcement learning agent chooses action by random in the first round. In the following rounds, reinforcement learning module is used. In this module Problem is a pair $(d c, c c)$, where $d c \in\{1,2, \ldots 25\}$ represent catch in a deep-sea area, and $c c \in\{1,2, \ldots, 15\}\}$ represents catch in a coastal area in the previous round. Answer is a triple representing ship allocation action $(h, d, c)$, such that $h, d, c \in\{0 \%, 25 \%, 50 \%, 75 \% 100 \%\}, d+c=1$. The Training data consists of a pair $\left(d c^{\prime}, c c^{\prime}\right)$, which is a catch in the current round, and a reward that is equal to the income (money earned by fishing decreased by ship maintenance costs). Learning strategy applied is the Q-Learning algorithm.

At the beginning $Q$ is initialized as a constant function 0 . To provide sufficient exploration, in a game number $g$ a random action is chosen with probability $1 / g$ (all actions have the same probability then). Therefore random or the best action (according to $Q$ function) is chosen and executed.

\subsection{Rule learning agent}

Because agent has no information what action is the best in the given situation, it is not able to prepare a training data in the form of (state, $\mathrm{f}($ state $)$ ). To overcome this problem the following work-around is used. Thank to comparison of income of all agents after action execution, the learning agent has information about quality of actions executed in the current situation. Leaning module is used to classify action in the given situation as good or bad. When it is learned, it may be used to give ranks to action candidates.

The Problem is defined as a five-tuple $(d c, c c, h, d, c)$, it consists of catch in the both areas during the previous round and a ship allocation action parameters. The Answer is an integer, which represents the given allocation action rating. The agent collects ratings for all generated allocation action candidates and chooses the action with the highest rating.

Training examples are generated from agent observations. Every round the learning agent stores ship allocations of all agents, and the fish catch in the previous round. The action of an agent with the highest income is classified as 


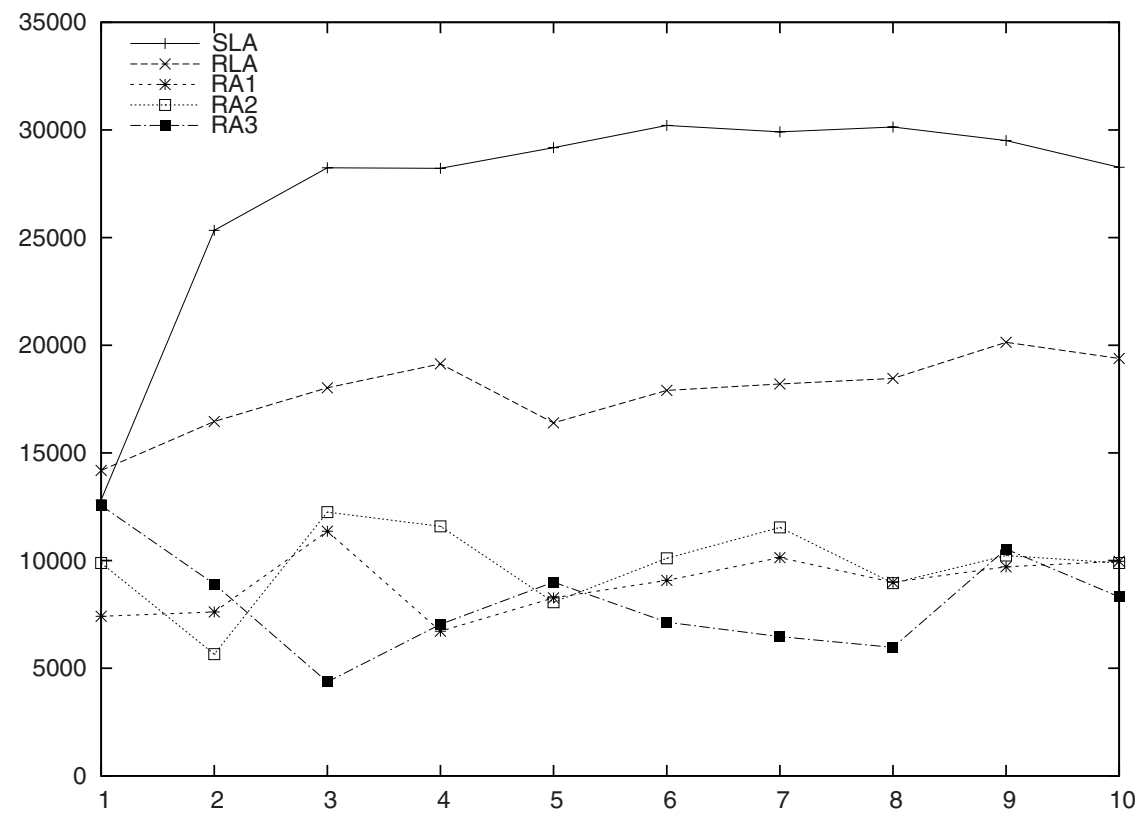

Fig. 2. The average performance of rule learning agent (SLA) reinforcement learning agent (RLA), and agents using random strategy (RA1, RA2, RA3)

good, and the action of an agent with the lowest income is classified as bad. If in some round all agents get the same income, none action is classified, and as a consequence, none of them is used in learning. Training data consists of the following pairs: $((d c, c c, h, d, c), q)$, where $q$ is equal to good or bad. At the end of each game the agent uses training examples, which were generated during all games played so far, to learn a new classifier, which is used in the next game. Learning strategy used is AQ21 program, which is an implementation of the AQ algorithm [13.

Rating $r$ of the action $a$ is calculated according to the formula:

$$
v(a)=\alpha \operatorname{good}(a)-\operatorname{bad}(a),
$$

where $\operatorname{good}(a)$ and $\operatorname{bad}(a)$ are numbers of rules, which match the action and current environment parameters, with consequence good and bad, respectively, and $\alpha$ is a weight representing the importance of rules with consequence good.

\subsection{Performance of the Agents}

The average performance of agents presented above in 10 subsequent games is presented in Fig. 2. In these experiments there were three random agents and one reinforcement learning agent or one rule learning agent ( $\alpha$ was equal to one). Performance was measured as a balance at the end of every game. In the figure the average performance from ten repetitions of the simulation is presented. 
In the experiments the average balance of both types of learning agents increases with time. Reinforcement learning agent was worse than a rule learning agent, but tuning of its parameters and taking into account during learning actions of other agents should increase its performance. On the other hand, reinforcement learning works well even if the reward is delayed. More about comparison of these two learning strategies can be found in [4].

\subsection{Two Learning Modules in One Agent}

Currently, we are working on the version of the system, in which agent will be learning in two aspects: ship allocation and setting a catch limit. The former aspect will be the same as described above. The latter will be used to develop a strategy of limiting fishing in the areas with small number of fish. Learning strategy that is assumed is Q-Learning algorithm. Currently, Problem is defined as a fish catch in the previous round, Answer contain information if the limit proposal (which is constant) should be accepted or not. Training data consists of the fish catch information and a reward. The reward is equal to 0 in all rounds except the last one, when it is a balance of the agent.

\section{Conclusion and Further Research}

In the paper an architecture of learning agent is proposed. It was used in the description of learning agents in a Fish-Banks simulation system. Agents are learning ship-allocation strategy using reinforcement learning and rule induction.

The architecture is general enough to represent different approaches to learning. Applying the proposed model in a description of the system makes the description clearer. It also helps to develop learning agents and to add new learning modules to existing agents. It may be considered as a tool for learning agents design.

Currently the architecture supports centralized learning only. In the future it should be extended to cover distributed learning (cooperation and communication during learning). Also agents with more then one learning module should be studied and the possibility of interaction between modules in the same agent should be examined.

Acknowledgments. The author is grateful to Arun Majumdar, Vivomind Intelligence Inc. for providing Prologix system (used for implementation), and for help with using it, Janusz Wojtusiak, MLI Laboratory for AQ21 software and assistance, and last but not least Jaroslaw Kozlak, AGH University of Science and Technology for help with the Fish Bank Game.

\section{References}

1. Sen, S., Weiss, G.: Learning in multiagent systems. In: Weiss, G. (ed.) A Modern Approach to Distributed Artificial Intelligence. MIT Press, Cambridge (1999)

2. Sugawara, T., Lesser, V.: On-line learning of coordination plans. In: Proceedings of the 12th International Workshop on Distributed Artificial Intelligence, pp. 335345, 371-377 (1993) 
3. Śnieżyński, K.J.: Learning in a multi-agent approach to a fish bank game. In: Pěchouček, M., Petta, P., Varga, L.Z. (eds.) CEEMAS 2005. LNCS (LNAI), vol. 3690, pp. 568-571. Springer, Heidelberg (2005)

4. Śnieżyński, B.: Resource Management in a Multi-agent System by Means of Reinforcement Learning and Supervised Rule Learning. In: Shi, Y., van Albada, G.D., Dongarra, J., Sloot, P.M.A. (eds.) ICCS 2007. LNCS, vol. 4488, pp. 864-871. Springer, Heidelberg (2007)

5. Russell, S., Norvig, P.: Artificial Intelligence - A Modern Approach. Prentice-Hall, Englewood Cliffs (1995)

6. Watkins, C.J.C.H.: Learning from Delayed Rewards. PhD thesis, King's College, Cambridge (1989)

7. Quinlan, J.: C4.5: Programs for Machine Learning. Morgan Kaufmann, San Francisco (1993)

8. Michalski, R.S., Larson, J.: Aqval/1 (aq7) user's guide and program description. Technical Report 731, Department of Computer Science, University of Illinois, Urbana (June 1975)

9. Sniezynski, B.: Rule induction in a fish bank multiagent system. Technical Report 1, AGH University of Science and Technology, Institute of Computer Science (2005)

10. Meadows, D., Iddman, T., Shannon, D.: Fish Banks, LTD: Game Administrator's Manual. Laboratory of Interactive Learning, University of New Hampshire, Durham, USA (1993)

11. Hardin, G.: The tragedy of commons. Science 162, 1243-1248 (1968)

12. Kozlak, J., Demazeau, Y., Bousquet, F.: Multi-agent system to model the fishbanks game process. In: The First International Workshop of Central and Eastern Europe on Multi-agent Systems (CEEMAS 1999), St. Petersburg (1999)

13. Wojtusiak, J.: AQ21 User's Guide. Reports of the Machine Learning and Inference Laboratory, MLI 04-3. George Mason University, Fairfax, VA (2004) 